\title{
Evaluation of the effectiveness of a clean delivery kit intervention in preventing cord infection and puerperal sepsis among neonates and their mothers in rural Mwanza Region, Tanzania
}

\author{
F. MOSHA ${ }^{*}$, S. WINANI ${ }^{2}$, S. WOOD $^{3}$, J. CHANGALUCHA $^{1} \&$ B. NGASALLA ${ }^{1}$ \\ ${ }^{1}$ National Institute for Medical Research, P.O. Box 1462, Mwanza, Tanzania \\ ${ }^{2}$ Regional Medical Office, Mwanza, Tanzania \\ ${ }^{3}$ Programme for Appropriate Technology in Health, Seattle, USA
}

\begin{abstract}
A study was carried out in Misungwi and Kwimba Districts, Tanzania to determine the effectiveness of clean delivery kits in preventing cord infection and puerperal sepsis and to provide qualitative information on community acceptability, correct use, and appropriateness of the kits. This study involved pregnant women aged 18-45 years old. In the delivery kit intervention population, the Maternal and Child Health Aide (MCHA) assigned to the health facility provided pregnant mothers with a clean delivery kit on their first antenatal visit. She explained how to use each of the kit components, with the aid of pictorial instructions included in the kit. The pregnant mothers were asked to convey the information to whoever assisted them during delivery. The MCHA also gave them health education based on the principles of the "six cleans" recognized by WHO (i.e., clean hands, clean perineum, clean delivery surface, clean cord cutting and tying instruments, clean cutting surface). Women received the clean delivery kit free of charge in accordance with the randomised stepped-wedge design schedule. During the first week following delivery, the Village Health Workers (VHWs) from both the intervention and control groups made two visits to the households of mothers who had delivered. They administered questionnaire about delivery to mother and birth attendant. During the two scheduled postpartum visits, those who were suspected to have puerperal sepsis or cord infection of the baby were referred to the health facility clinician for confirmation. Results indicated that use of clean delivery kit had a positive effect on reducing both cord infection and puerperal sepsis. The use of a clean home delivery kit coupled with an educational intervention about the "six cleans" had a significant effect on reducing the incidence of cord infection and puerperal sepsis among women enrolled in the study. In low resource settings where home birth is common and clean delivery supplies are scarce, disposable kits can be made available through health clinics, markets, pharmacies or other channels to help reduce rates of infection.
\end{abstract}

Key words: delivery, neonates, mothers, cord infection, puerperal sepsis, Tanzania

\section{Introduction}

Tetanus and other infections are leading causes of neonatal deaths. Worldwide some 500,000 infants die each year of neonatal tetanus, and a further 460,000 die as a consequence of severe bacterial infection (WHO, 1998). Cord infections contribute significantly to neonatal infection. For instance, a study in India found that, in $47 \%$ of infants hospitalized with sepsis, cord infection was the source; and $21 \%$ of infants admitted for other reasons had cord infection.

The reasons for the high incidence of cord infection in the developing world are the high proportion of home deliveries, unhygienic cutting of the cord, application of unclean substances to the cord stump, and covering the stump with unclean fabric. Strategies to reduce the risk of neonatal tetanus and cord infection include promoting clean delivery and clean cord care as well as increasing tetanus toxoid immunization.

Puerperal sepsis, attributed to pregnancy complications is responsible for $5-20 \%$ of all maternal deaths worldwide (WHO, 1992) and causes the loss of more than one third of healthy years of life (WDR, 1993). In sub-Saharan Africa, the incidence of puerperal sepsis is very high. A study in an urban hospital in Nairobi, Kenya, found the incidence to be $20 \%$, (Plummer et al., 1987) and in Mwanza Region of Tanzania puerperal sepsis causes $17 \%$ of all maternal deaths (MMCH, 1999) A major cause of puerperal sepsis is unhygienic delivery (Gibbs, 1980). In Tanzania, 53\% of all deliveries take place at home in unhygienic conditions (TDHS, 1996). During such deliveries, unclean substances are often introduced into the

\footnotetext{
* Correspondence: Frank Mosha; Email: fmosha2002@yahoo.co.uk
} 
vagina (unclean hands, local herbs, etc.), unclean delivery surface is often used, and unclean cloth is used to cover the vagina after delivery (Gibbs, 1980). Likewise, the cord is frequently cut by unclean instruments, and unclean substances are put on the cord (Gibbs, 1980). These practices increase the likelihood of microorganisms entering the birth canal of the woman or the cord stump of the neonate, causing puerperal sepsis or cord infection, respectively.

Clean delivery kits have been developed by UNFPA, UNICEF, and other international and local organizations in order to help ensure hygienic deliveries and cord care. In sub-Saharan Africa, the effectiveness of these kits in preventing puerperal sepsis and cord infections has never been evaluated. Similarly, very few qualitative or behavioural research studies in these communities have been done to determine acceptability, correct use, and appropriateness of these kits.

\section{Materials and Methods}

This study was implemented among pregnant women aged 18 - 45 years old in eight wards in Kwimba and Missungwi Districts in Mwanza Region, north-western Tanzania.

It was a stepped-wedge randomised community trial. The study sites were sectioned into ten clusters; each cluster was defined as a dispensary or health centre providing antenatal care to its service population. The ten clusters (eight dispensaries and two health centres plus their service areas) were based on an implementation schedule of one new cluster every six weeks. The cluster implementation schedule was randomised.

In the delivery kit intervention population, the Maternal and Child Health Aide (MCHA) assigned to the health facility provided pregnant mothers with a clean delivery kit on their first antenatal visit. She explained how to use each of the kit components, with the aid of pictorial instructions included in the kit. The delivery kit contained a plastic sheet, soap, clean razor blade, cord ties and user instructions. The aide was instructed by the investigators to ask the women to convey the information to whoever assisted them during delivery. The MCHA also provided the pregnant women with health education based on the principles of the "six cleans" recognized by the World Health Organization: clean hands, clean perineum, clean delivery surface, clean cord cutting and tying instruments, clean cutting surface.

Eighteen in-depth interviews were conducted randomly across the clusters. Ten were kit users (1 had puerperal sepsis, 9 without infection) and 8 were kit non-users (2 had puerperal sepsis, 1 with cord infection, 5 without infection).

\section{Results}

The results were based on 3,262 women aged 18-45 years old. Their marital status being 68\% monogamous, $16 \%$ polygamous, $12 \%$ single and $4 \%$ divorced or widowed. Sixty-seven percent had completed 7 years of primary education, $13 \%$ did

Table 1: Adjusted odds ratio of factors that are associated with cord infection

\begin{tabular}{llll}
\hline Risk Factor & Odds ratio & $P$-value & $\begin{array}{l}\text { CI for odds } \\
\text { ratio }\end{array}$ \\
\hline $\begin{array}{l}\text { Kit use } \\
\text { Yes }\end{array}$ & 1.0 & & \\
$\quad$ No & 12.6 & $<0.001$ & $4.96-32.10$ \\
Bath before delivery & 1.0 & & \\
$\quad$ Yes & 3.4 & $<0.001$ & $1.77-6.53$ \\
$\quad$ No & 1.0 & & \\
$\begin{array}{l}\text { Shave before delivery } \\
\text { Yes }\end{array}$ & 0.96 & 0.923 & $0.42-2.18$ \\
$\quad$ No & 1.0 & & \\
$\begin{array}{l}\text { Place of delivery } \\
\text { Home }\end{array}$ & 1.0 & 0.890 & $0.58-1.86$ \\
$\quad \begin{array}{l}\text { Health facility } \\
\quad \text { Other places }\end{array}$ & 1.2 & 0.789 & $0.28-5.40$ \\
Substances put on cord & & & \\
$\quad \begin{array}{l}\text { Nothing } \\
\text { Some substance }\end{array}$ & 1.0 & 0.201 & $0.78-3.30$ \\
\hline
\end{tabular}


not complete primary education, $2 \%$ had secondary school education, and $18 \%$ had never been to school. Multiple logistic regression was conducted with variables that were significantly associated with both kit use and puerperal sepsis or cord infection. the vulva area that could be introduced into the birth canal before, during or after delivery, or introduced into the cord during cutting. Information about use of the soap in the kit by the women to wash themselves before delivery was not collected. However, the presence of the soap in the kit may

Table 2: Adjusted odds ratio of risk factors that are associated with puerperal sepsis

\begin{tabular}{|c|c|c|c|}
\hline Risk Factor & Odds ratio & $P$-value & $\begin{array}{l}\mathrm{CI} \text { for odds } \\
\text { ratio }\end{array}$ \\
\hline \multicolumn{4}{|l|}{ Kit use } \\
\hline Yes & 1.0 & & \\
\hline No & 3.4 & $<0.001$ & $1.94-5.86$ \\
\hline \multicolumn{4}{|l|}{ Bath before delivery } \\
\hline Yes & 1.0 & & \\
\hline No & 2.7 & $<0.001$ & $1.59-4.70$ \\
\hline \multicolumn{4}{|l|}{ Place of delivery } \\
\hline Home & 1.0 & & \\
\hline Health facility & 0.7 & 0.169 & $0.40-1.17$ \\
\hline Other places & 1.7 & 0.349 & $0.57-4.87$ \\
\hline \multicolumn{4}{|l|}{ Duration of labour } \\
\hline $0-3 \mathrm{hrs}^{*}$ & 1.0 & & \\
\hline $3-6 \mathrm{hrs}$ & 1.3 & 0.441 & $0.66-2.61$ \\
\hline$>6 \mathrm{hrs}$ & 2.7 & 0.004 & $1.37-5.50$ \\
\hline
\end{tabular}

Results indicated that the use of the clean delivery kits had a positive effect on reducing both cord infection and puerperal sepsis. The effect of the kit on reducing cord infection was much greater than on puerperal sepsis, but both were significant. Data from the in-depth interview showed that, there was high acceptability of the delivery kits by women in rural Mwanza.

Our findings suggest that the kit was perceived as appropriate for women in rural areas. They did not report difficulties in using it and they recommended it to be used by other women. Respondents who used the kit reported satisfaction with the way the kit contributed to a clean delivery. All respondents showed willingness to pay for the delivery kit.

\section{Discussion}

Taking bath and using soap before delivery decreases the incidence of puerperal sepsis and cord infection by reducing the number of exogenous organisms on the body surface, in the perineum and have made it easier for women to take bath before delivery.

The concept of "six cleans" as advocated by WHO (1998) reiterates the importance of clean hands, clean delivery surface, clean perineum, nothing unclean inserted into the vagina, clean cord cutting tool and clean cord tie during delivery. Each of the kit contents are selected to help assure one of these "six cleans". For the bar of soap provided the means of hygiene required to achieve clean hands and a clean perineum. The plastic sheet provided the means to achieve a clean delivery surface. The razor blade and cord ties in the delivery kit provide a clean cord-cutting surface and clean cutting and tying instruments.

Washing hands by birth attendants before the delivery and the use of sterile gloves was not found to be an important factor affecting rates of sepsis. While sterile gloves used by trained health workers reduce contamination, the technique of wearing gloves is not always easy for home birth attendants or family members to master and thus the use of gloves does not guarantee sterility. Re-use of gloves could also potentially be a problem. 
Longer labour (more than six hours) was also associated with increased risk of puerperal sepsis although it was not retained in the final logistic regression model. This was not unexpected as women with longer duration of labour are more likely to experience long hours between the rapture of membranes and delivery, more hours of uterine contraction, and an increase in ascending spread of exogenous and endogenous microorganisms that can cause upper genital tract infection.

The use of both maternal recall (aided by photos) and direct interviewer observations, coupled with confirmation from the clinician, improved the likelihood that all infection present in the study population was identified. The short time maternal recall due to the timely nature of health worker visits at three and five days after delivery helped assure that the information was reliable.

It should be noted, however, that the study was implemented in two districts where a non-profit organisation has managed a system for active community-based maternal and perinatal surveillance for several years. It is possible that women living in these districts had more exposure to information about clean delivery than women in other areas of Tanzania. One component of the organisation's surveillance system was to teach mothers about clean delivery and cord care. During the study, mothers in both the intervention and control areas were taught about the "six cleans", with mothers in the intervention areas also being given a clean delivery kit.

This study showed that women who used the kit and had ancillary education had better outcomes than women who did not use the kit, but still received ancillary education. It is likely that the delivery kit plus ancillary education intervention would have shown an even greater effect in an area where the population was not exposed to an ancillary education intervention on the six cleans. It is possible that without this ancillary education, the impact of the use of clean delivery kits in preventing puerperal sepsis and cord infection would be greater.

In conclusion the use of clean delivery kit coupled with an educational intervention about the "six cleans" has a significant effect on reducing the incidence of cord infection in neonates and puerperal sepsis among women. In low resource settings where a high proportion of mothers give birth at home, supplies needed to conduct a clean delivery are often not available even in rural dispensaries and health centres. Making clean delivery kit available through health clinics, markets, pharmacies or other commercial channels will likely help reduce rates of infection.

The qualitative research indicated clean home delivery kits are viewed as acceptable, appropriate, and useful items, and that women are willing to pay a small fee for them. Kits should therefore, be made widely available at health facilities, pharmacies and possibly, shops. Training health care workers may be an effective way of educating women about the usefulness of clean delivery kits. Comprehensive maternal and newborn health programmes that include training of skilled birth attendants are particularly appropriate venues in which to introduce clean delivery kits.

\section{References}

Gibbs, R.S. (1980) Clinical risk factors for puerperal infection. Obstetrics and Gynaecology, 55, (5 suppl ) S178-S183.

MMCH (1999) Mwanza Maternal and Child Health Annual Report, Mwanza, Tanzania.

Plummer, F.A., Laga, M., Brunham, R.C., Piot, P., Ronald, A.R., Bhullar, V., Mati, J.Y., Ndinya-Achola, J.O., Cheang, M., \& Nsanze, H. (1987) Postpartum upper genital tract infections in Nairobi, Kenya: Epidemiology, etiology and risk factors. Journal of Infectious Disease, 156, 92-98.

TDHS (1996) Tanzania Demographic and Health Survey, Dar es Salaam, Tanzania.

WHO (1992) The Prevention and Management of Puerperal Infections. Report of a technical working group. 20-22 May, 1992, Geneva World Health Organization, (WHO/FHE//95.4)

WHO (1998) Care of the umbilical cord: a review the evidence. Chapter 1, page 1 (WHO/RHTMS/98.4).

WDR (1993) World Development Report: Investing in Health. 